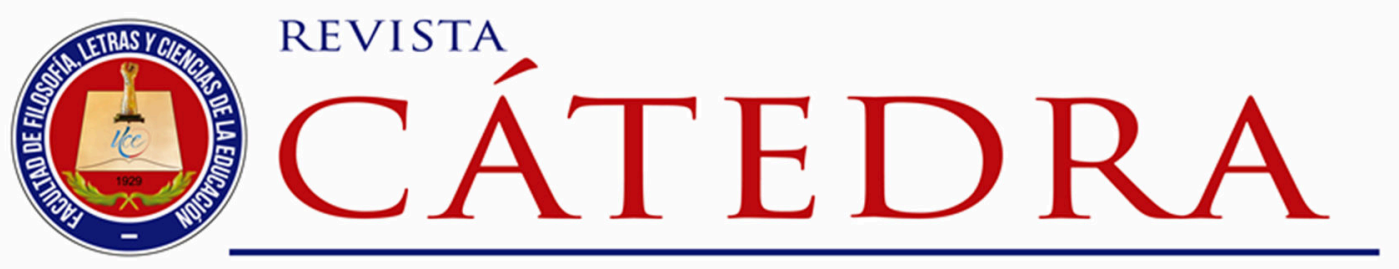

\title{
Metodologías de la enseñanza-aprendizaje en la educación virtual
}

\section{Teaching-learning methodologies in virtual education}

\author{
José Daniel Espinosa-Rodríguez \\ Universidad Andina Simón Bolívar, Quito, Ecuador \\ jose.espinosa@uasb.edu.ec \\ https://orcid.org/0000-0002-6457-4922
}

(Recibido: 14/11/2021; Aceptado: 18/11/2021; Versión final recibida: 22/12/2021)

Cita del artículo: Espinosa-Rodríguez, J.D. (2022). Metodologías de la enseñanzaaprendizaje en la educación virtual. Revista Cátedra, 5(1), 19-31.

\section{Resumen}

El aprendizaje es un proceso continuo y diverso que requiere conocimientos y competencias para afrontar la vida y hacerles frente a los retos del siglo XXI, los cuales se agudizaron debido a la emergencia sanitaria derivada por la covid-19. En ese sentido, la implementación de metodologías activas en entornos virtuales de aprendizaje (EVA), son una respuesta para superar los retos presentados en cuanto al desarrollo de aprendizajes significativos en modalidades no presenciales y a la innovación de la práctica pedagógica mediadas por las Tecnologías del Aprendizaje y el Conocimiento (TAC). Entre las metodologías más utilizadas en los EVA se encuentran el método de casos, el aprendizaje basado en problemas, el aprendizaje basado en proyectos y el aula invertida. Adicionalmente, existen técnicas que permiten "activar" momentos de aprendizaje, fomentando la participación, el trabajo colaborativo y la generación de contenidos de los estudiantes, privilegiando así la aplicación por sobre la transmisión de contenidos. Por otro lado, para introducir de forma efectiva las TAC en el proceso de enseñanza-aprendizaje a través de las metodologías activas supone transformar la práctica educativa desde el cambio de roles (docente y estudiantes) y su relación con el dominio digital, a través del desarrollo de competencias digitales. Finalmente, existen una gran cantidad de recursos digitales que pueden ser utilizados como parte de las metodologías activas, cuyo objetivo será superar el rol pasivo de estudiantes y docentes como consumidores de información, permitiéndoles adoptar un rol más activo en la generación de nuevos conocimientos a partir del intercambio con otros usuarios de la web.

Palabras clave

Aprendizaje, enseñanza, entornos virtuales de aprendizaje, metodologías activas, TAC. 


\section{Abstract}

Learning is a continuous and diverse process that requires knowledge and competencies to face life and the challenges of the 21st century, which became more acute due to the health emergency caused by Covid-19. In this sense, the implementation of active methodologies in virtual learning environments (VLE) are a response to overcome the challenges presented in terms of the development of meaningful learning in non-face-to-face modalities and the innovation of pedagogical practice mediated by CT. Among the most commonly used methodologies in VLE are the case method, problem-based learning, project-based learning and the inverted classroom. In addition, there are techniques that allow "activating" learning moments, encouraging participation, collaborative work and the generation of content by students, thus favoring application over transmission of content. On the other hand, to effectively introduce ICT in the teaching-learning process through active methodologies involves transforming the educational practice from the change of roles (teacher and students) and their relationship with the digital domain, through the development of digital competencies. Finally, there are a large number of digital resources that can be used as part of active methodologies, whose objective will be to overcome the passive role of students and teachers as consumers of information, allowing them to adopt a more active role in the generation of new knowledge from the exchange with other web users.

\section{Keywords}

Learning, teaching, active methodologies, LKT, virtual learning environment.

\section{Introducción}

La situación mundial derivada de la crisis sanitaria por la pandemia de la covid-19, ha sido un detonante para replantear, y en muchos casos reafirmar, la necesidad de un cambio para la transformación eficiente en el ámbito educativo. Según el informe de la UNESCO-IESALC (2020), alrededor de 166 países tomaron la decisión de suspender actividades presenciales en sus instituciones educativas de nivel básico, medio y superior, con el propósito de salvaguardar a la población estudiantil y a toda la comunidad educativa. En ese orden de ideas, a nivel mundial se estima que el $87 \%$ de la población estudiantil se vio perjudicada por estas medidas; esto supone alrededor de unos 1,520 millones de estudiantes afectados.

Como consecuencia de lo descrito anteriormente, los sistemas educativos se vieron en la necesidad de utilizar espacios no presenciales y entornos digitales de aprendizaje como alternativas para continuar ofertando los servicios educativos y la implementación de los procesos de enseñanza-aprendizaje. En el contexto actual derivado de esta pandemia se ha evidenciado ciertas carencias de las instituciones educativas, sobre todo en relación con la infraestructura y a la formación del personal docente en cuanto al conocimiento y aplicación de modalidades no presenciales (Alcántara Santuario, 2020) Sin embargo, también ha abierto un área de oportunidad para poner en marcha estrategias de mejoras, particularmente en lo referente con el interés por la utilización de recursos y estrategias que permitan facilitar la docencia a través de los entornos digitales. A pesar de los esfuerzos antes mencionados, hay que reconocer que no siempre la respuesta fue la más adecuada tanto de docentes como de estudiantes respecto al uso eficiente de herramientas digitales y al desarrollo de las competencias digitales, pero sí en un tiempo récord (García-Peñalvo et al., 2020)

Tomando como base lo descrito en líneas atrás, otros aspectos que han inquietado a la comunidad educativa derivados de los retos presentados en la implementación de las modalidades no presenciales se encuentran, por un lado, aquellos que están estrechamente vinculados al desarrollo de aprendizajes en modalidad virtual, es decir, garantizar 
aprendizajes significativos con ayuda de la tecnología; y por otro, la posibilidad de innovar la práctica pedagógica, considerando la adecuación de los resultados de aprendizaje, objetivos, procesos y métodos de enseñanza-aprendizaje ante la nueva situación, modificando significativamente lo que hasta el momento se hacía, sin alterar el proyecto educativo.

Ante este panorama, quizá una de las respuestas más favorable para lograr superar los retos presentados en cuanto al desarrollo de aprendizajes en modalidades no presenciales y a la innovación de la práctica pedagógica, se centra en la implementación de metodologías activas de aprendizaje mediadas por las $\mathrm{TAC}^{1}$, las cuales distan por mucho de la virtualización de clases magistrales (que en muchos casos ha supuesto la solución de urgencia ante la emergencia sanitaria tal como lo muestran estudios recientes, (Tejedor et al., 2020).

Siguiendo este orden de ideas, el presente trabajo trata de dar un perspectiva en relación con el aprendizaje y las metodologías activas que, de acuerdo con su finalidad y diseño, pueden ser aplicadas en espacios y entornos virtuales de aprendizaje y que permiten, por un lado, un adecuado proceso de enseñanza-aprendizaje, así como una mediación más eficiente de la práctica docente a través del uso de la tecnología; y por otro, la generación de competencias digitales tanto de docentes como de estudiantes, las cuales suponen un manejo adecuado de las TAC cuyo propósito es aprovechar al máximo los recursos y herramientas disponibles para la interacción y creación de conocimientos y asimilación de aprendizajes.

\section{El aprendizaje en el contexto de las metodologías activas}

Hablar de aprendizaje en estos tiempos supone reconocerlo como un proceso multidimensional de la condición humana donde convergen distintas formas de llevarlo a cabo, con estilos singulares, donde interactúan una amplia variedad de contenidos y a distintas profundidades, así como en contextos diferenciados. En ese sentido, el aprendizaje involucra el accionar de diferentes actores quienes se comunican según el rol del momento, pudiéndolo modificar o intercambiar entre ellos; esto supone entonces que la interconexión que hay entre los actores, de acuerdo con Herrera-Pavo et. al (2021), "está dada por actividades desarrolladas de manera conjunta que permiten que las y los estudiantes pueden asimilar, analizar y ejercitar lo aprendido a través de experiencias concretas y significativas para su contexto cercano" (Herrera-Pavo et al., 2021, p. 15). Dichos autores también comentan que estas actividades se desarrollan a través de la mediación docente y la comunidad de aprendizaje en modalidades presenciales y no convencionales.

Lo anterior indican los autores supone entonces que el proceso de aprendizaje a través de estas actividades "no hace referencia a la presencia física de los actores (docentes y estudiantes), sino al tipo de actividad donde estos actores trabajan colaborativamente el uno con el otro, de manera que su accionar sólo se entienda y cobre significado en el marco de alcanzar un objetivo común." (Herrera-Pavo et al., 2021, p. 15).

Para implementar las actividades de manera conjunta y lograr los resultados esperados es necesario emplear aquellas metodologías que hacen referencia al aprendizaje basado en la indagación o comúnmente denominadas metodologías activas. Tomando como base la idea del párrafo anterior, cuando se habla de metodologías activas tiene sentido hablar del

1 En el contexto educativo las TAC -Tecnologías del Aprendizaje y el Conocimiento- tratan de reconducir las TIC -Tecnologías de la Información y Comunicación- hacia un uso más formativo y pedagógico (Velasco-Rodríguez, 2017).

Licencia Creative Commons Atribución 4.0 Internacional (CC BY 4.0)

Revista Cátedra, 5(1), pp. 19-31, enero-junio 2022. e-ISSN: 2631-2875

https://doi.org/10.29166/catedra.v5i1.3435 
aprendizaje activo como un proceso dinámico donde se busca que los estudiantes puedan tener experiencias auténticas y que desarrollen procesos metacognitivos de manera autónoma y colaborativa. Aplicando estos aspectos, los estudiantes, con una adecuada mediación docente, serán quienes determinan el ritmo y estilo de asimilación y generación de nuevo conocimiento a partir de sus conocimientos previos.

En ese orden de ideas y de acuerdo con el informe de la Asociación para el Estudio de la Educación Superior Norteamericana (Bonwell y Eison, 1991), el cual señala que hay ciertas características asociadas a la utilización de estrategias que promueven el aprendizaje activo, tales como: a) los estudiantes hacen más que solo escuchar, se implican en un proceso de pensamiento de orden superior y se implican en actividades colaborativas; b) la transmisión de información se enfatiza menos y se da espacio para el desarrollo de las capacidades de los estudiantes, así como se enfatiza la exploración de actitudes y valores de los estudiantes.

Por tanto, y siguiendo el planteamiento de estos autores, se podría indicar que el aprendizaje activo es un aprendizaje "que implica a los estudiantes en el hacer y en la reflexión sobre lo que están haciendo" (Bonwell y Eison, 1991, p. 45), elementos fundamentales para el proceso de enseñanza-aprendizaje mediados por las TAC, del cual se profundizará más adelante.

Como se ha señalado en párrafos anteriores, las metodologías activas de enseñanzaaprendizaje tienen la finalidad de maximizar las probabilidades de que los estudiantes aprendan bajo situaciones donde cobren sentido los contextos y escenarios propuestos por el docente de manera intencionada. Por tanto, para lograr la finalidad es necesario tomar en cuenta algunos principios que permitan diseñar actividades a partir de dichas situaciones bajo las metodologías activas.

Para ello las investigaciones de Ambrose (2010) son una guía clara sobre el alcance que tienen dichos principios del aprendizaje quienes a su vez lo reconocen como un proceso de desarrollo que se interseca con otros procesos de desarrollo en la vida del estudiante, los cuales poseen además de habilidades, conocimientos y destrezas; también experiencias sociales y emocionales que influencian lo que valoran, su autopercepción y la de los otros, y formas para involucrase en el proceso de aprendizaje. Estos principios se articulan entre sí y en situaciones reales de aprendizaje, además de ser indivisibles, por lo que una manera fácil de entender estos principios y las buenas prácticas docentes es la asociación que proponen Espejo y Sarmiento (2017) para orientar la implementación pertinente de ciertas metodologías activas. Dicho esto, a continuación, se enuncian los principios y sus buenas prácticas en el cuadro 1.

\begin{tabular}{ll}
\hline \multicolumn{1}{c}{ Principio } & \multicolumn{1}{c}{ Ejemplo de buena práctica } \\
\hline El conocimiento previo & $\begin{array}{l}\text { Realizar un diagnóstico utilizando diferentes actividades o } \\
\text { aplicando diferentes procedimientos. }\end{array}$ \\
\hline $\begin{array}{l}\text { La forma en que los } \\
\text { estudiantes organizan } \\
\text { el conocimiento }\end{array}$ & $\begin{array}{l}\text { Utilizar organizadores gráficos - por ejemplo, un mapa } \\
\text { conceptual - tanto al inicio como a lo largo del curso (por } \\
\text { ejemplo, para realizar un resumen de cada unidad). }\end{array}$ \\
\hline $\begin{array}{l}\text { La motivación de los } \\
\text { estudiantes }\end{array}$ & $\begin{array}{l}\text { Utilizar un test de motivación al inicio, al medio y al final de un } \\
\text { curso. }\end{array}$ \\
$\begin{array}{l}\text { Para desarrollar } \\
\text { dominio sobre un } \\
\text { tema, los estudiantes } \\
\text { deben desarrollar }\end{array}$ & $\begin{array}{l}\text { Identificar claramente los recursos asociados a las } \\
\text { competencias del perfil de egreso y asociarlos a los resultados } \\
\text { de aprendizaje del curso. }\end{array}$ \\
\hline
\end{tabular}

Licencia Creative Commons Atribución 4.0 Internacional (CC BY 4.0) 
recursos cognitivos, actitudinales y procedimentales
Evaluar considerando no solo los aspectos cognitivos o procedimentales, sino también lo actitudinal y lo metacognitivo.

Utilizar variados procedimientos evaluativos entre otros, escalas de apreciación y rúbricas.

Una práctica orientada por metas y acoplada con retroalimentación focalizada potencia la calidad del aprendizaje de los estudiantes

El nivel actual de desarrollo de los estudiantes interactúa con el clima social, emocional e intelectual de la clase

Para ser aprendices autónomos, los estudiantes deben aprender a monitorear y ajustar su forma de enfrentar el aprendizaje
Socializar los resultados de aprendizaje del curso con los estudiantes y especificarlos en una guía de aprendizaje o sílabo.

Generar un sistema de retroalimentación que dé información al estudiante respecto del avance en su proceso de aprendizaje.

Utilizar técnicas de "rompe hielo" al inicio del curso y a lo largo de éste.

Preguntar a los estudiantes cómo se sienten con relación al curso.

Utilizar técnicas de trabajo en grupo que permitan el desarrollo de lazos sociales entre los estudiantes.

Establecer un sistema que permita a los estudiantes chequear su avance en el curso.

Socializar y discutir con los estudiantes la carga de trabajo autónomo asociado al curso.

Discutir con los estudiantes las técnicas de estudio más adaptadas a la materia del curso. Intercambiar experiencias entre los estudiantes.

Cuadro 1. Aplicación de los principios del aprendizaje de Susan Ambrose. Fuente: (Espejo y Sarmiento, 2017, p. 14)

\subsection{Metodologías Activas}

Tal como se ha descrito en el acápite anterior, las metodologías activas son necesarias para integrarse en el proceso de enseñanza-aprendizaje, el cual pueda trascender de una reproducción del conocimiento a una dinámica participativa de los actores involucrados dentro de todo el proceso educativo. Tomando en cuenta tanto actividades de aprendizaje auténticas, estrategias de acompañamiento y seguimiento, así como evidencias claras y con sentido respecto de la calidad de lo que se espera enseñar, así como las interacciones efectivas para lograr los objetivos y demostrar así los resultados de aprendizaje.

Lo anterior como parte de una educación de calidad simulando así lo que demanda hoy en día la sociedad, es decir, individuos con habilidades tales como: autonomía, desarrollo del trabajo en pequeños equipos multidisciplinares, actitud participativa, habilidades de comunicación y cooperación, resolución de problemas, creatividad, etc., aspectos que tienen que estar contemplados en la educación del siglo XXI.

Ahora bien, con la incorporación e investigación continua de las metodologías activas, se esperaría un cambio adecuado de las prácticas docentes, no solo en lo que respecta a una visión diferente del propósito de la educación, sino ofrecer herramientas metodológicas con las que puedan tanto los docentes como otros profesionales de la educación, implementar procesos mucho más creativos, reflexivos y con una sólida base de conocimientos técnicos 
y tecnológicos, con el objeto de ser capaces de transferir todos estos componentes a los estudiantes para que estos últimos puedan seguir aprendiendo a lo largo de la vida.

Bajo ese escenario es importante indicar que las metodologías activas, responden al paradigma socio-constructivista; lo que ubica al proceso de aprendizaje en la búsqueda de la emancipación de los individuos, combinando altas dosis de autonomía con procesos equilibrados de rendición de cuentas que permitan que los estudiantes se hagan responsables de sus aprendizajes. Tomando como referencia a Barkley y Cross (2007) y a Espejo y Sarmiento (2017) las metodologías activas se pueden clasificar en dos grandes grupos, la primera requiere del desarrollo de habilidades de planificación, priorización, organización, comunicación y movilización de conocimientos, así como el componente metacognitivo del proceso de aprendizaje individual de los estudiantes, esta última dada la necesidad de reflexionar sobre cada etapa del trabajo que realizan, evaluando sus resultados personales y los de su grupo. Por lo que estas requieren de un trabajo continuo en el tiempo. Entre esta clasificación se encuentran: a) el método de casos, b) aprendizaje basado en problemas, c) aprendizaje basado en proyectos y d) aula invertida.

Para una mejor comprensión y a manera de bosquejar en qué consisten cada metodología, a continuación, en el cuadro 2 se presenta un extracto del trabajo de Espejo y Sarmiento (2017)donde se presenta una breve síntesis de los aspectos más relevantes de cada una.

\begin{tabular}{|c|c|c|c|c|}
\hline Metodología & En qué consiste? & $\begin{array}{l}\text { ¿Cuándo } \\
\text { utilizarlo? }\end{array}$ & Tiempo & Evaluación \\
\hline de & $\begin{array}{l}\text { Los estudiantes } \\
\text { deben ser capaces } \\
\text { de identificar los } \\
\text { aspectos clave de } \\
\text { la información que } \\
\text { se presenta como } \\
\text { caso-problema, así } \\
\text { como determinar } \\
\text { un conjunto de } \\
\text { soluciones } \\
\text { posibles, proponer } \\
\text { estrategias para su } \\
\text { solución y tomar } \\
\text { decisiones frente a } \\
\text { las problemáticas } \\
\text { asociadas. }\end{array}$ & $\begin{array}{l}\text { Puede ser } \\
\text { implementada } \\
\text { con estudiantes } \\
\text { de cualquier año } \\
\text { ajustando los } \\
\text { casos propuestos } \\
\text { retroalimentando } \\
\text { de manera } \\
\text { adecuada el } \\
\text { trabajo } \\
\text { individual y } \\
\text { grupal. }\end{array}$ & $\begin{array}{l}\text { Se } \\
\text { recomienda } \\
\text { ocuparla } \\
\text { dentro de } \\
\text { toda una } \\
\text { unidad } \\
\text { temática. }\end{array}$ & $\begin{array}{l}\text { Se realiza a } \\
\text { través de los } \\
\text { resultados de } \\
\text { las propuestas } \\
\text { de solución del } \\
\text { caso tanto } \\
\text { individual } \\
\text { como grupal. } \\
\text { Se requiere de } \\
\text { un instrumento } \\
\text { a base de } \\
\text { criterios } \\
\text { (rúbrica o lista } \\
\text { de cotejo) }\end{array}$ \\
\hline $\begin{array}{l}\text { Aprendizaje } \\
\text { basado en } \\
\text { problemas }\end{array}$ & $\begin{array}{l}\text { Consiste en dirigir } \\
\text { al estudiante hacia } \\
\text { el aprendizaje a } \\
\text { través de un } \\
\text { problema; es decir, } \\
\text { antes de que los } \\
\text { estudiantes } \\
\text { adquieran un } \\
\text { determinado } \\
\text { conocimiento se } \\
\text { les ofrece un } \\
\text { problema para } \\
\text { que, a través de él, } \\
\text { descubran qué }\end{array}$ & $\begin{array}{l}\text { Para su } \\
\text { implementación, } \\
\text { es importante } \\
\text { considerar el } \\
\text { nivel y } \\
\text { características de } \\
\text { los estudiantes } \\
\text { con el fin de } \\
\text { escoger } \\
\text { problemas que } \\
\text { sean } \\
\text { comprensibles } \\
\text { por ellos. Por } \\
\text { esta razón esta } \\
\text { metodología }\end{array}$ & $\begin{array}{l}\text { Se sugiere } \\
\text { que el } \\
\text { profesor } \\
\text { destine un } \\
\text { cierto } \\
\text { número de } \\
\text { horas } \\
\text { semanales al } \\
\text { trabajo de } \\
\text { los } \\
\text { estudiantes, } \\
\text { de manera } \\
\text { que esté } \\
\text { presente y } \\
\text { pueda }\end{array}$ & $\begin{array}{l}\text { Se realiza a } \\
\text { través de la } \\
\text { entrega de } \\
\text { informes } \\
\text { preliminares al } \\
\text { terminar cada } \\
\text { una de las } \\
\text { siguiente } \\
\text { etapas: a) } \\
\text { análisis del } \\
\text { problema y } \\
\text { determinación } \\
\text { de los } \\
\text { conocimientos } \\
\text { requeridos }\end{array}$ \\
\hline
\end{tabular}




\begin{tabular}{|c|c|c|c|c|}
\hline & $\begin{array}{l}\text { necesitan saber } \\
\text { para resolverlo. }\end{array}$ & $\begin{array}{l}\text { tiene a funcionar } \\
\text { mejor con } \\
\text { estudiantes que } \\
\text { ya tienen una } \\
\text { cierta base en la } \\
\text { disciplina de } \\
\text { estudio. }\end{array}$ & $\begin{array}{l}\text { acompañar } \\
\text { el proceso. }\end{array}$ & $\begin{array}{l}\text { para resolverlo, } \\
\text { b) Resultado de } \\
\text { la investigación } \\
\text { sobre } \\
\text { conocimientos } \\
\text { necesarios y c) } \\
\text { Integración } \\
\text { final y } \\
\text { propuesta de } \\
\text { solución. Al } \\
\text { finalizar el } \\
\text { proceso, el } \\
\text { profesor puede } \\
\text { incentivar la } \\
\text { hetero, co y } \\
\text { autoevaluación } \\
\text { a través de } \\
\text { rúbricas } \\
\text { preparadas } \\
\text { para este } \\
\text { efecto. }\end{array}$ \\
\hline $\begin{array}{l}\text { Aprendizaje } \\
\text { basado en } \\
\text { proyectos }\end{array}$ & $\begin{array}{l}\text { Se lleva a cabo a } \\
\text { partir del trabajo } \\
\text { en grupos de } \\
\text { estudiantes, } \\
\text { quienes eligen un } \\
\text { tema de acuerdo } \\
\text { con sus intereses y } \\
\text { elaboran un } \\
\text { proyecto } \\
\text { relacionado. El } \\
\text { grupo de trabajo } \\
\text { tiene la autonomía } \\
\text { necesaria para } \\
\text { establecer sus } \\
\text { objetivos, su } \\
\text { planificación y } \\
\text { tomar decisiones, } \\
\text { teniendo el tiempo } \\
\text { necesario para } \\
\text { reflexionar sobre } \\
\text { sus acciones y } \\
\text { orientar su trabajo. }\end{array}$ & $\begin{array}{l}\text { Se utiliza } \\
\text { generalmente } \\
\text { con estudiantes } \\
\text { de años } \\
\text { superiores, en } \\
\text { estricto rigor } \\
\text { puede ser } \\
\text { adaptada para } \\
\text { estudiantes de } \\
\text { cualquier año } \\
\text { ajustando los } \\
\text { temas } \\
\text { propuestos y el } \\
\text { grado de } \\
\text { autonomía que se } \\
\text { requiere para el } \\
\text { buen desarrollo } \\
\text { del proyecto. }\end{array}$ & $\begin{array}{l}\text { Se } \\
\text { recomienda } \\
\text { ocupar } \\
\text { horas a lo } \\
\text { largo de un } \\
\text { periodo } \\
\text { académico. } \\
\text { Es posible } \\
\text { utilizar } \\
\text { tiempos más } \\
\text { cortos, pero } \\
\text { se requerirá } \\
\text { ajustar la } \\
\text { planificación } \\
\text { de manera } \\
\text { que sea } \\
\text { factible para } \\
\text { los } \\
\text { estudiantes. }\end{array}$ & $\begin{array}{l}\text { Se realiza a } \\
\text { través de las } \\
\text { entregas } \\
\text { preliminares y } \\
\text { la entrega final } \\
\text { que han sido } \\
\text { acordadas. Es } \\
\text { importante } \\
\text { incluir también } \\
\text { la co y } \\
\text { autoevaluación } \\
\text { en relación con } \\
\text { el trabajo en } \\
\text { general y a la } \\
\text { dinámica del } \\
\text { grupo en } \\
\text { particular. }\end{array}$ \\
\hline $\begin{array}{l}\text { Aula } \\
\text { invertida }\end{array}$ & $\begin{array}{l}\text { Esta considera la } \\
\text { definición de un } \\
\text { conjunto de } \\
\text { competencias que } \\
\text { serán } \\
\text { desarrolladas por } \\
\text { los estudiantes, } \\
\text { transfiriendo, por } \\
\text { un lado, la }\end{array}$ & $\begin{array}{l}\text { Esta puede ser } \\
\text { aplicada en todas } \\
\text { las áreas } \\
\text { curriculares, así } \\
\text { como dentro de } \\
\text { los diferentes } \\
\text { niveles de } \\
\text { educación: } \\
\text { básica, básica }\end{array}$ & $\begin{array}{l}\text { Se } \\
\text { recomienda } \\
\text { ocupar esta } \\
\text { metodología } \\
\text { a lo largo del } \\
\text { ciclo, } \\
\text { periodo o } \\
\text { módulo una }\end{array}$ & 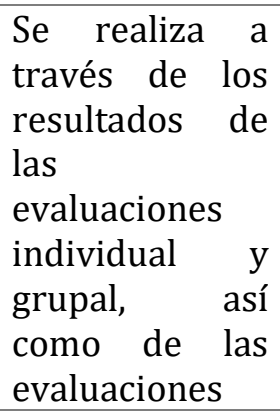 \\
\hline
\end{tabular}




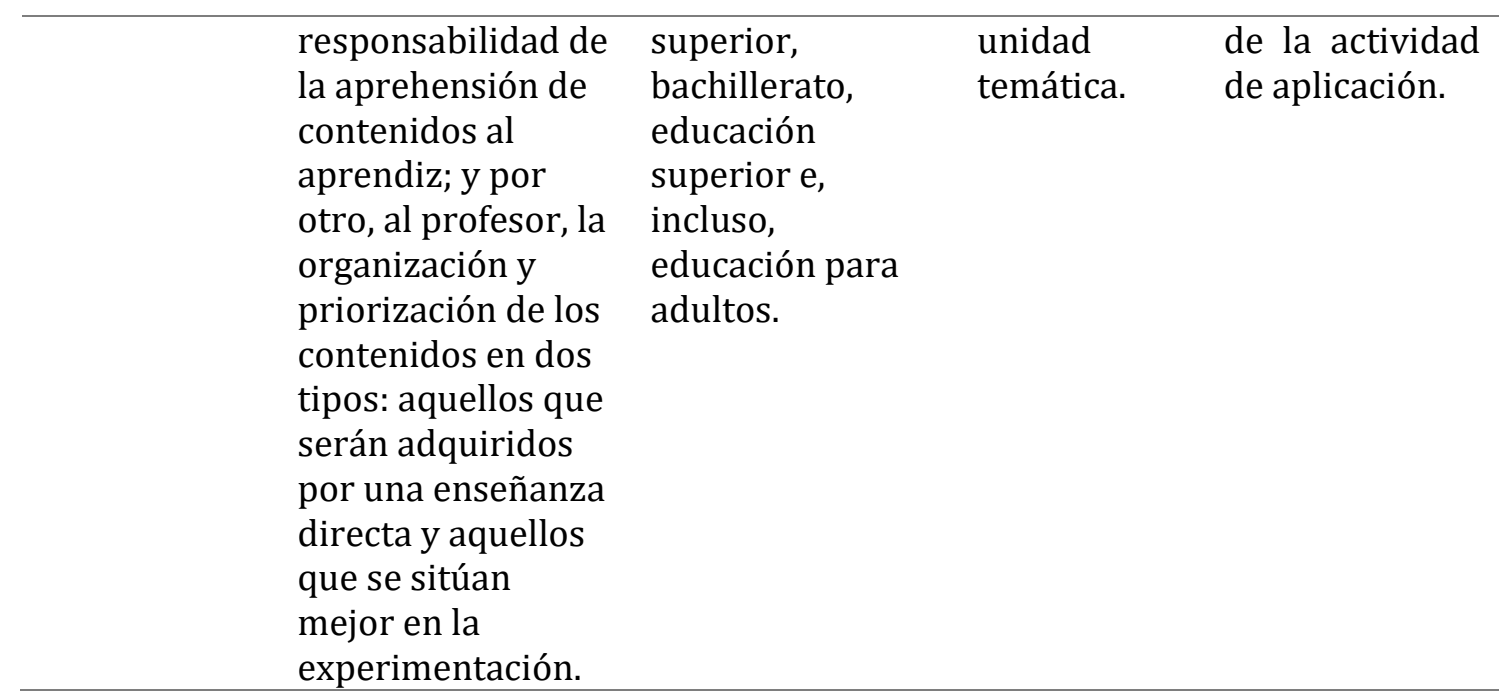

Cuadro 2. Metodologías para utilizar a lo largo de una unidad didáctica. Fuente: (Espejo y Sarmiento, 2017, pp. 47-67)

Por otra parte, la segunda clasificación, aunque no son propiamente metodologías sino técnicas específicas, permiten "activar" un momento de aprendizaje, fomentando la participación, el trabajo colaborativo y la aplicación de contenidos de los estudiantes. Estas, a diferencia de las anteriores, solo requieren un tiempo específico para su implementación. Para los fines de este trabajo, es importante mencionar que este grupo de técnicas permite conectarse con los aprendizajes mediados por las TAC, los cuales abordaremos en el siguiente acápite.

Adicionalmente hay que indicar que su propósito es aprovechar al máximo el tiempo de contacto directo con los estudiantes privilegiando así actividades de aplicación por sobre la transmisión de contenidos. Entre ellas se encuentran las técnicas: a) para fomentar la discusión en la clase, b) de enseñanza recíproca, c) que utilizan organizadores gráficos y, d) centradas en la escritura.

\section{El aprendizaje mediado por las TAC}

El término TAC, hace referencia al uso que se hace de las Tecnologías de la Información y Comunicación (TIC) desde una dimensión más formativa y pedagógica. De este modo y de acuerdo con Lozano (2011) "las TAC van más allá de aprender meramente a usar las TIC y apuestan por explorar estas herramientas tecnológicas al servicio del aprendizaje y de la adquisición del conocimiento" (Lozano, 2011, párr. 7).

Por ello, para introducir de forma efectiva las TAC en el proceso de enseñanza-aprendizaje supone transformar la práctica educativa desde el cambio de roles (docente y estudiantes) y la relación de estos actores con el uso y dominio digital, a través del desarrollo de competencias digitales.

Lo anterior tiene como consecuencia visualizar la práctica docente como una oportunidad de cambio y mejora, ya que, por un lado, las estrategias implementadas en situaciones convencionales ya no son satisfactorias para los nuevos escenarios donde se privilegia el uso de entornos digitales. Lo que conlleva a la redefinición del rol docente en relación con las actividades de aprendizaje, tareas, conocimientos, actitudes y la formación de competencias, las cuales hoy en día, están mediadas por la tecnología. Por tanto, el docente "debe dejar de ser un instructor que domina los conocimientos, para convertirse en asesor, orientador, facilitador y mediador del proceso de enseñanza-aprendizaje" (Segura et al., 2007, p. 6) 
Por otro lado, el rol del estudiantado también se ve trastocado por el uso de herramientas digitales, ya que implica una mayor motivación en el aprendizaje (Area Moreira, 2010; Pedró, 2011), así como una actitud diferente frente a la utilización de dicha tecnología, la cual enriquece el aprendizaje y contribuye a ganar confianza en sí mismo (Pedró, 2011).

Sin embargo, tal como lo comenta Salinas (2004), estos recursos educativos necesitan ser dominados para la construcción del conocimiento; en ese sentido, los estudiantes requieren de nuevas destrezas para afrontar esta realidad. Por tanto, el rol de los estudiantes ya no se ciñe a un simple reproductor de contenidos, sino que "debe llegar a ser un usuario inteligente y crítico de la información, para lo que precisa aprender a buscar, obtener, procesar y comunicar información y convertirla en conocimiento" (Segura et al., 2007, p. 6). En sintonía con lo descrito en los párrafos anteriores, es necesario analizar el contexto educativo y tecnológico en el que se desempeña la práctica docente. Esto quiere decir que se debe considerar las características de los estudiantes, sus capacidades, destrezas y competencias a desarrollar, los recursos disponibles, entre otros. Lo anterior con el propósito de decidir entre aquellas metodologías, técnicas activas y recursos TAC que les permita a los estudiantes adquirir conocimientos a partir de los diferentes estilos de aprendizaje que hayan desarrollado. Es decir, permitirles ser protagonistas de sus propios aprendizajes mediados por la tecnología y entornos digitales.

Ahora bien, la incorporación de las TAC en los diferentes escenarios y contextos educativos tiene, además de los propósitos ya mencionados en líneas previas, superar el rol pasivo tanto de estudiantes y docentes como consumidores de información, permitiéndoles adoptar un rol más activo y permitirles posicionarse como creadores de contenido y generadores de nuevos conocimientos a partir de la generación e intercambio con otros usuarios de la web.

\subsection{Tipos de Tecnologías del Aprendizaje y el Conocimiento de acuerdo con su propósito}

De acuerdo con Velasco-Rodríguez (2017) existen un gran número de recursos TAC con los que los estudiantes pueden crear diferentes contenidos y generar un aprendizaje significativo. Estos recursos les permite ser los protagonistas y responsables de su proceso de enseñanza-aprendizaje, además a partir de la mediación del docente, la cual consiste en la presentación, colaboración, supervisión y acompañamiento en el uso de las TAC se puede aprovechar un mayor potencial.

En ese sentido, este autor presenta un conjunto de TAC que, de acuerdo con su propósito pueden ser utilizados como parte de las estrategias de aprendizaje en los entonos digitales de aprendizaje, comúnmente también llamados EVA. Por lo que a continuación se presentan en el cuadro 3 el propósito, los recursos y una breve descripción de las TAC.

\begin{tabular}{lll}
\hline \multicolumn{1}{c}{ Propósito } & \multicolumn{1}{c}{ Recurso TAC } & \multicolumn{1}{c}{ Descripción } \\
\hline Edición vídeo & $\begin{array}{l}\text { Quik, Splice, Stupeflix y 123 } \\
\text { apps }\end{array}$ & $\begin{array}{l}\text { crear y editar vídeos de una manera } \\
\text { sencilla }\end{array}$ \\
\hline $\begin{array}{l}\text { Vídeos } \\
\text { interactivos }\end{array}$ & Vizia y EDpuzzle & $\begin{array}{l}\text { convertir cualquier vídeo en una } \\
\text { lección interactiva }\end{array}$ \\
\hline Audio & $\begin{array}{l}\text { SoundCloud, Audacity, Peggo } \\
\text { y Vocaroor archivos audio y poder } \\
\text { compartirlos }\end{array}$ \\
\hline $\begin{array}{l}\text { Imagen - } \\
\text { infografías }\end{array}$ & $\begin{array}{l}\text { Genially, Typorama, Fotojet y } \\
\text { creación de contenido gráfico estático } \\
\text { o dinámico con objetivos diversos }\end{array}$ \\
\hline $\begin{array}{l}\text { Gestión del } \\
\text { aula }\end{array}$ & $\begin{array}{l}\text { Moodle, Blackboard, Canvas, } \\
\text { Edmodo, Classroom, Doceo y } \\
\text { Additio }\end{array}$ & $\begin{array}{l}\text { gestionar el día a día de un docente: } \\
\text { listas, evaluación, rúbricas, banco de } \\
\text { recursos, organización de grupos, etc. }\end{array}$ \\
\hline
\end{tabular}

Licencia Creative Commons Atribución 4.0 Internacional (CC BY 4.0)

Revista Cátedra, 5(1), pp. 19-31, enero-junio 2022. e-ISSN: 2631-2875

https://doi.org/10.29166/catedra.v5i1.3435 


\begin{tabular}{|c|c|c|}
\hline Gamificación & $\begin{array}{l}\text { Cerebriti Edu, Quizizz, } \\
\text { Trivinet, Jeopardy Rocks, } \\
\text { Playbuzz, Triventy y } \\
\text { Arcademic }\end{array}$ & $\begin{array}{l}\text { Generar actividades interactivas } \\
\text { utilizando recursos lúdicos para los } \\
\text { contenidos curriculares }\end{array}$ \\
\hline Evaluación & $\begin{array}{l}\text { Apester, Kahoot!, Google } \\
\text { Forms y Quiz Revolution }\end{array}$ & $\begin{array}{l}\text { comprobar los aprendizajes } \\
\text { adquiridos a través de recursos e } \\
\text { instrumentos de evaluación }\end{array}$ \\
\hline Comunicación & Homeroom, Otter y Blogger & $\begin{array}{l}\text { establecer una comunicación fluida } \\
\text { entre todos los miembros de la } \\
\text { comunidad educativa }\end{array}$ \\
\hline Presentaciones & $\begin{array}{l}\text { Emaze, Genially, Powtoon, } \\
\text { Prezi, Sparkol y Haiku Deck, }\end{array}$ & $\begin{array}{l}\text { hacer presentaciones de una manera } \\
\text { sencilla y con unos resultados } \\
\text { atractivos y profesionales }\end{array}$ \\
\hline $\begin{array}{l}\text { Generadores de } \\
\text { actividades }\end{array}$ & Educaplay y Kubbu & $\begin{array}{l}\text { crear actividades interactivas propias } \\
\text { de manera personalizada }\end{array}$ \\
\hline $\begin{array}{l}\text { Trabajo } \\
\text { colaborativo }\end{array}$ & $\begin{array}{l}\text { Padlet, Team Maker, } \\
\text { Teamweek, Meetingwords y } \\
\text { Quip }\end{array}$ & $\begin{array}{l}\text { crear de recursos y herramientas } \\
\text { didácticas de trabajo compartido }\end{array}$ \\
\hline $\begin{array}{l}\text { Buscadores y } \\
\text { navegación } \\
\text { segura }\end{array}$ & YouTube Kids y Kiddle & $\begin{array}{l}\text { buscar vídeos e información con total } \\
\text { seguridad }\end{array}$ \\
\hline $\begin{array}{l}\text { Derechos de } \\
\text { autor }\end{array}$ & $\begin{array}{l}\text { Pixabay, Iconicons, } \\
\text { Skitterphoto, Sample Focus y } \\
\text { pngimg }\end{array}$ & $\begin{array}{l}\text { buscar imágenes, archivos audio, } \\
\text { iconos, etc. sin derechos de autor para } \\
\text { utilizar en nuestros proyectos }\end{array}$ \\
\hline $\begin{array}{l}\text { Mapas } \\
\text { mentales }\end{array}$ & Coggle y WiseMapping & crear mapas mentales digitales \\
\hline Idiomas & $\begin{array}{l}\text { Lyrics training, Locallingual } \\
\text { y Forvo }\end{array}$ & $\begin{array}{l}\text { aprender idiomas a través de audios } \\
\text { reales y de situaciones interactivas }\end{array}$ \\
\hline Producciones & Pixton, Tiki toki y Storybird & $\begin{array}{l}\text { crear cómics, líneas del tiempo o } \\
\text { cuentos }\end{array}$ \\
\hline
\end{tabular}

Cuadro 3. TAC de acuerdo con su propósito. Fuente: (Velasco-Rodríguez, 2017, pp. 774-775)

Tomando en cuenta lo anterior, se puede evidenciar que el uso de las TAC permite por un lado un cambio de paradigma en la escuela y hacia una enseñanza de calidad, donde la motivación y la mejora de los resultados esperados se logren (Pedró, 2011). Y por el otro, la posibilidad de que las tecnologías trasciendan de un elemento meramente instrumental a potenciar un modelo educativo actual y eficiente, mismo que responda a las necesidades formativas de los ciudadanos" (Castañeda-Quintero y Adell-Segura, 2013, p. 45).

\section{Conclusiones}

Como conclusiones se podría indicar que el aprendizaje, al ser un proceso continuo y diverso, permite a los seres humanos adquirir conocimientos y competencias necesarias para afrontar la vida y ser capaces de hacer frente a los retos del siglo XXI. De acuerdo con esta idea se debe reconocer que el aprendizaje hoy en día se construye no solo en las aulas de clase sino por fuera de las instituciones de cualquier nivel educativo; sin embargo, el rol que ha adquirido en estos últimos años las instituciones de educación es reinterpretar los conocimientos y los aprendizajes de forma activa e interconectada a partir del diálogo pedagógico entre el estudiante, los saberes, la tecnología y el docente (De Zubiría Samper, 2006, p. 2)

Bajo ese escenario, se esperaría que la enseñanza-aprendizaje trascienda de una reproducción del conocimiento a una dinámica participativa de los actores involucrados 
dentro de todo el proceso educativo; donde se tome en cuenta tanto metodologías activas donde se puedan establecer actividades de aprendizaje, estrategias de acompañamiento y seguimiento, así como el desarrollo de las competencias digitales para aprovechar al máximo la aplicación de las TAC con las cuales se motive a los estudiantes a potenciar su creatividad e incrementar sus habilidades multitarea, así como aprovechar las sinergias entre docentes y estudiantes a través de los entornos virtuales de aprendizaje. 


\section{Bibliografía}

Alcántara Santuario, A. (2020). Educación superior y COVID-19: Una perspectiva comparada. En H. Casanova Cardiel, Educación y pandemia: Una visión académica (pp. 75-82). Instituto de Investigaciones sobre la Universidad y la Educación. http://132.248.192.241:8080/jspui/bitstream/IISUE_UNAM/541/1/AlcantaraA_2 020_Educacion_superior_y_covid.pdf

Ambrose, S. A. (2010). How Learning Works: Seven Research-Based Principles for Smart

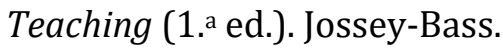

Area Moreira, M. (2010). El proceso de integración y uso pedagógico de las TIC en los centros educativos: Un estudio de casos. Revista de educación, 352, 77-97.

Barkley, E., y Cross, P. (2007). Tecnicas de aprendizaje colaborativo: Manual para el profesorado universitario. Morata. http://ticuah.weebly.com/uploads/9/6/4/6/9646574/barkley__aprendizaje_colaborativo.pdf

Bonwell, C. C., y Eison, J. A. (1991). Active Learning: Creating Excitement in the Classroom. 1991 ASHE-ERIC Higher Education Reports. ERIC Clearinghouse on Higher Education, The George Washington University, One Dupont Circle, Suite 630, Washington, DC 20036-1183 (\$17. https://eric.ed.gov/?id=ED336049

Castañeda Quintero, L. J., y Adell Segura, J. (2013). La anatomía de los PLEs. En Entornos personales de aprendizaje: Claves para el ecosistema educativo en red, 2013, ISBN (p. 11). Editorial Marfil. https://dialnet.unirioja.es/servlet/articulo?codigo=7453547

De Zubiría Samper, J. (2006). Los modelos pedagógicos. En Hacia una Pedagogía Dialogante (segunda, p. 250). Aulla Abierta.

Espejo, R., y Sarmiento, R. (Eds.). (2017). Metodologías activas para el aprendizaje. Universidad Central de Chile.

García-Peñalvo, F. J., Corell, A., Abella-García, V., y Grande, M. (2020). La evaluación online en la educación superior en tiempos de la COVID-19. Education in the Knowledge Society (EKS), 21, 26-26. https://doi.org/10.14201/eks.23086

Herrera-Pavo, M. Á., Espinoza-Weaver, Y., Rivera Bilbao- la Vieja, G. M., Espinosa-Rodríguez, J. D., y Orellana-Navarrete, V. (2021). Ruta pedagógica hacia el 2030: La propuesta de un modelo para el -sistema educativo ecuatoriano. Revista Andina de Educación, 4(1), 1-4. https://doi.org/10.32719/26312816.2020.4.1.0

Lozano, R. (2011). De las TIC a las TAC: Tecnologías del aprendizaje y del conocimiento. Anuario ThinkEPI, 5(1), 45-47.

Pedró, F. (2011). Tecnología y escuela. Lo que funciona y por qué. Fundación Santillana. https://www.researchgate.net/publication/234139384_Tecnologia_y_escuela_Lo_ que_funciona_y_por_que

Salinas, J. (2004). Innovación docente y uso de las TIC en la enseñanza universitaria. RUSC. Universities and Knowledge Society Journal, 1(1), Article 1. https://doi.org/10.7238/rusc.v1i1.228

Segura, M., Candioti, C., y Medina, C. J. (2007). LAS TECNOLOGÍAS DE LA INFORMACIÓN Y LA COMUNICACIÓN (TIC) EN LA EDUCACIÓN: RETOS Y POSIBILIDADES (XXII Semana Monográfica de la Educación, p. 185). Fundación Santillana. https://www.fundacionsantillana.com/PDFs/xxii_semana_monografica.pdf

Tejedor, S., Cervi, L., Pérez-Escoda, A., y TUsa, F. (2020). Digital Literacy and Higher Education during COVID-19 Lockdown: Spain, Italy, and Ecuador. Publications, 8, 117. https://doi.org/10.3390/publications8040048

UNESCO-IESALC. (2020). COVID-19 y educación superior: De los efectos inmediatos al día después: Análisis de impactos, respuestas políticas y recomendaciones (p. 57). Organización de las Naciones Unidas para la Educación, la Ciencia y la Cultura e Instituto Internacional de la UNESCO para la Educación Superior en América Latina $y$ el Caribe. https://www.iesalc.unesco.org/2020/04/14/iesalc-insta-a-los- 
estados-a-asegurar-el-derecho-a-la-educacion-superior-en-igualdad-deoportunidades-ante-el-covid-19/

Velasco-Rodríguez, M. Á. (2017). Las TAC y los recursos para generar aprendizaje. Revista $\begin{array}{llll}\text { Infancia, Educación } \quad \text { y } & \text { Aprendizaje, }\end{array}$ https://doi.org/10.22370/ieya.2017.3.2.796

\section{Autor}

JOSÉ DANIEL ESPINOSA-RODRÍGUEZ obtuvo su título de Magíster en Investigación en Educación por la Universidad Andina Simón Bolívar (Ecuador) en 2017. Obtuvo el título de Licenciado en Pedagogía por la Universidad Nacional Autónoma de México en 2010.

Actualmente es Coordinador del Centro de Evaluación e Innovación Educativa de la Universidad UTE, Ecuador. Profesor invitado de posgrado en el Área de Educación de la Universidad Andina Simón Bolívar. Es articulista de la Revista Tsafiqui - Revista Científica en Ciencias Sociales de la Universidad UTE (Ecuador). Articulista y miembro del Consejo Internacional de Revisores de Revista Andina de Educación de la Universidad Andina Simón Bolívar (Ecuador). Sus principales temas de investigación incluyen la evaluación educativa en educación básica, media y superior; Desarrollo de competencias digitales docentes, formación docente para el desarrollo de metodologías activas para la enseñanza en educación superior. 